\title{
Endoscopic submucosal dissection for colorectal laterally spreading tumors with the Dual knife only
}

\author{
Danxia He, Xinguang Cao, Te Luo, Wenpeng Tao, Changqing Guo \\ Department of Gastroenterology, the First Affiliated Hospital of Zhengzhou University, Zhengzhou, China
}

Videosurgery Miniinv 2019; 14 (4): 495-500

DOI: https://doi.org/10.5114/wiitm.2019.84217

\begin{abstract}
Introduction: The knives for endoscopic submucosal dissection (ESD) have their strengths as well as shortcomings. They need to be used in combination in most cases. The Dual knife is a relatively novel type of ESD knife produced in 2009, which can be used for completing the whole procedure of ESD.

Aim: Colorectal laterally spreading tumors (LSTS) are a special subtype of colorectal neoplasms. We aimed to evaluate the clinical features and outcomes of ESD for colorectal LSTs only using the Dual knife from our experience.

Material and methods: This retrospective study included 162 patients (each patient had 1 lesion) with colorectal LSTs treated by ESD with the Dual knife by a single endoscopist at our hospital between June 2015 and January 2018. We analyzed the clinical features and outcomes after resection.

Results: We obtained the en bloc ESD of the colorectal LSTS and the complete histological assessment in all patients. The mean age of the patients was 53.9 years. The mean diameter of lesions was $46 \mathrm{~mm}$. The most common location of LSTs was the rectum. The most common histological type was tubular adenoma with 63 cases. The mean operating time was $56 \mathrm{~min}$. Perforation and bleeding rates were $0.6 \%$ and $0.6 \%$, respectively. No cases of local persistence or recurrence were observed at a follow-up endoscopy 6 to 31 months after the en bloc resection.

Conclusions: The ESD using the Dual knife only for colorectal LSTs has the advantages of safety, efficiency, and minimally invasiveness. It is worthy of widespread clinical application.
\end{abstract}

Key words: colorectal laterally spreading tumors, Dual knife, endoscopic submucosal dissection.

\section{Introduction}

Colorectal laterally spreading tumors (LSTs) were first described in 1993 [1]. The lesions represent a special subtype of colorectal neoplasms, which are defined as more than $10 \mathrm{~mm}$ in diameter, and grow laterally and circumferentially typically rather than vertically along the wall of the colorectum [2]. The LSTs are subdivided into granular (LST-G) and non-granular (LST-NG) types based on the endoscopic finding, and the granular type consists of homogeneous (LST-G-H) or nodular-mixed (LST-G-M) morphology while the non-granular type is flat-elevated
(LST-NG-F) or pseudo-depressed (LST-NG-PD) [3]. The LSTs have been recognized as a significant precursor of advanced colorectal cancer (CRC) [4]. In addition, LSTs are prone to be overlooked in colonoscopy because of the unique morphological features, and are technically challenging in endoscopic resection (ER), which probably causes incomplete en bloc resection and local recurrence. Hence, the endoscopic diagnosis and treatment of LSTs are greatly essential for colorectal cancer prevention. To date, ER, including endoscopic mucosal resection (EMR) and endoscopic submucosal dissection (ESD), has been indicated for the lesions. The ESD has many predominant ad-

\section{Address for correspondence}

Changqing Guo, Department of Gastroenterology, the First Affiliated Hospital of Zhengzhou University, 1 Jianshe Road, Erqi District,

450052 Zhengzhou, China, phone: +86 3716627 1127, e-mail: 1807872771@qq.com 
vantages over EMR for large lesions [5]. A variety of high-frequency electric knives have been developed for ESD, such as the Hook knife [6], insulation-tipped diathermic knife (IT knife) [7], Flush knife [8], Flex knife [9], bipolar needle knife (B-knife) [10], SB knife [11], and Triangle tip knife (TT knife) [12], etc. The various knives have their strengths as well as shortcomings. They need to be used in combination in most cases. The Dual knife is a relatively novel type of ESD knife produced in 2009, which can be used for completing the whole procedure of ESD.

We report herein the results of a clinical study in which only the Dual knife was used for the ESD procedure of colorectal LSTs.

\section{Aim}

Colorectal LSTs are a special subtype of colorectal neoplasms. We aimed to evaluate the clinical features and outcomes of ESD for colorectal LSTs only using the Dual knife from our experience.

\section{Material and methods}

\section{Participants}

The study subjects were 162 patients (each patient had 1 lesion) with colorectal LSTs treated by ESD (only using the Dual knife) by a single endoscopist at our hospital between June 2015 and January 2018. The study protocol was conducted in accordance with the tenets of the revised Declaration of Helsinki (1983) and was approved by the ethics committee of our institution. Informed consent was obtained from all the patients.

\section{Bowel preparation}

Bowel preparation involved a patient drinking 2-3 I of polyethylene glycol (PEG) electrolyte solution before the procedure, then an assessment of the quality of bowel cleanliness on the basis of the Boston Bowel Preparation Scale (BBPS); a BBPS score $\geq 5$ was satisfactory [13].

\section{Settings of colorectal ESD}

All procedures were performed using a colonoscope (CF-H260; Olympus, Japan) with a transparent cap (D-201-13404; Olympus, Japan) and a gastroscope (GIF-H260); Olympus, Japan) with a distal attachment (ND-201-11804; Olympus, Japan). A Dual knife (KD-650Q; Olympus, Japan) was used during all ESD procedures. A high-frequency electrosurgical generator (VIO 200S; ERBE, Germany) was set up. An injection needle (NM-200L-0423; Olympus, Japan) was used to lift up the lesion. Hot biopsy forceps (FD-1U-1; Olympus, Japan) or endoscopic metal clips (HX-610-090L; Olympus, Japan) were used to stop bleeding. A mixture of $100 \mathrm{ml}$ of $10 \%$ glycerol solution containing $2 \mathrm{ml}$ of methylthioninium chloride and $1 \mathrm{mg}$ of $0.002 \%$ epinephrine was used as the injection solution [14].

\section{The ESD procedures}

The ESD was carried out as follows: 1) Marking: It could assist to indicate the margin of lesions during the procedure. However, marking was not usually used in the colorectal LSTs because the range of lesions was generally clear (Photo 1 A). 2) Submucosal injection: the injection solution mentioned above is locally injected into the submucosa of a lesion through the injection needle (Photo 1 B). An additional submucosal injection was repeated as needed. 3) Incising the mucosa: After submucosal injection, the mucosal layer around the lesion (3-5 mm outside lesion edge) was incised circumferentially using a Dual knife with an endo-cut Q mode (effect 3, duration 2, interval 2) (Photo 1 C). 4) Dissecting the submucosa: The submucosal layer beneath the lesion was then dissected using the Dual knife with a force-coagulation mode (effect 3, $40 \mathrm{~W}$ ). 5) Treatment of the post-ESD resection site: careful examination and coagulation of exposed visible vessels (with little or no overlying submucosa) in the post-ESD site with hot biopsy forceps (Photo 1 D). Endoscopic metal clips were used for suturing the resection bed as appropriate. Finally, the lesion was resected completely and retrieved endoscopically.

\section{Management after ESD}

All patients routinely underwent the post-operative hospital stay, the fasting period and infusion. A reasonable diet was given after confirming the absence of inflammatory findings, such as fever, abdominal pain, and level of serum C-reactive protein (CRP), while inspecting delayed bleeding and delayed perforation. Both the length of the fasting period and hospitalization should be considered with respect to each specific situation. All the patients with colorectal ESD received a follow-up endosco- 

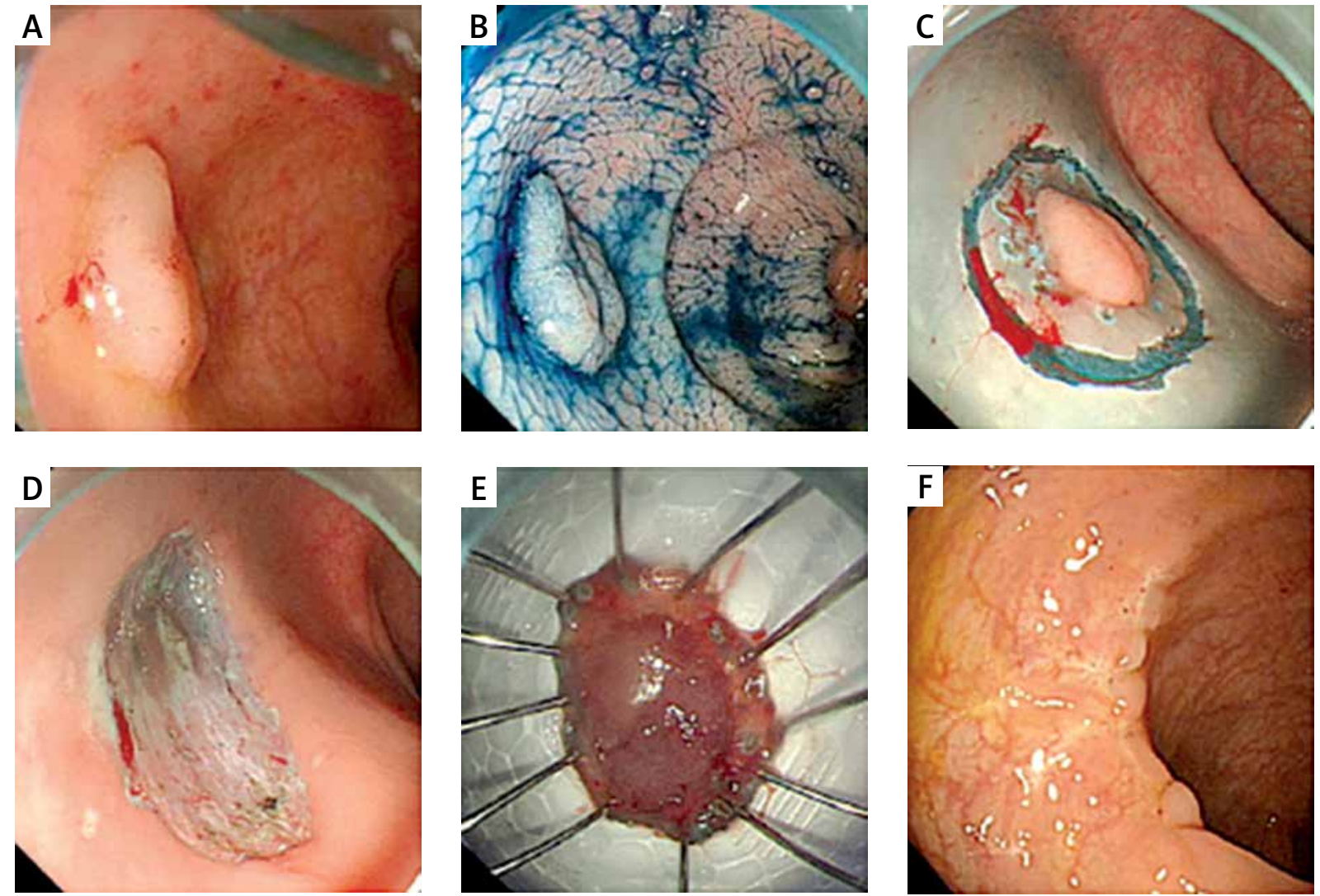

Photo 1. Endoscopic submucosal dissection (ESD) procedures, performed with a Dual knife. A - A $20 \mathrm{~mm}$ flat-elevated nongranular type of laterally spreading tumor (LST-NG-F) located in the rectum. B - Lesion margins were delineated with $0.4 \%$ indigo-carmine spraying before ESD. C - Following injection of $10 \%$ glycerol solution containing methylthioninium chloride and $0.002 \%$ epinephrine into the submucosal layer, a circumferential incision was made using the Dual knife. D - An ESD was then carried out using the Dual knife. The ulcer bed is represented here after the successful en bloc resection. $\mathbf{E}-$ The resected specimen was $20 \mathrm{~mm} \times 20 \mathrm{~mm}$ in diameter and histology revealed a tubular adenoma. F - Follow-up endoscopy 6 months after ESD showed no local persistence or recurrence

py 6 to 31 months after the resection to check for any local recurrence on the basis of endoscopic and pathological assessments (Photo $1 \mathrm{~F}$ ).

\section{Histopathology}

Resected specimens were pinned flat on the corkboard with sufficient tension (Photo $1 \mathrm{E}$ ). Subsequently, the specimens were immersed in 10\% formalin, embedded in paraffin, serially sectioned into pieces at intervals of $2 \mathrm{~mm}$, and stained with hematoxylin and eosin. Finally, the following parameters - the histopathological type, resection margins (lateral and basal) of the lesions, and the invasive depth of the lesions - were evaluated by an expert pathologist with regard to the Vienna classification of gastrointestinal epithelial neoplasia [15].

\section{Variables investigated}

The variables were investigated as follows: patients' clinical features (including sex and age), tumor location, tumor size, pathologic type, LST subtypes, ESD procedure times, en bloc rate and complications (including bleeding and perforation). The local recurrence rate after complete en bloc resection was also assessed.

\section{Results}

A total of 162 patients with colorectal LSTs treated by ESD using the Dual knife were enrolled in the study. The clinicopathological characteristics and outcome of ESD treatment for LSTs in this study are presented in Table I. The mean age of the patients 
Table I. Clinicopathological features and outcomes of ESD treatment for colorectal LSTs with Dual knife only in this study

\begin{tabular}{|c|c|}
\hline Parameter & Result \\
\hline Age, mean (range) [years] & $53.9(24-81)$ \\
\hline Sex (male/female) & $93 / 69(1.35: 1)$ \\
\hline Tumor diameter, mean (range) [mm] & $46(12-100)$ \\
\hline \multicolumn{2}{|l|}{ LST types and subtypes, $n(\%)$ : } \\
\hline Granular: & $118(72.8)$ \\
\hline Homogeneous & $61(37.7)$ \\
\hline Nodular-mixed & $57(35.2)$ \\
\hline Non-granular: & $44(27.2)$ \\
\hline Flat-elevated & $39(24.1)$ \\
\hline Pseudo-depressed & $5(3.1)$ \\
\hline \multicolumn{2}{|l|}{ Tumor location, $n(\%)$ : } \\
\hline Cecum & $5(3.1)$ \\
\hline Ascending colon & $11(6.8)$ \\
\hline Transverse colon & $17(10.5)$ \\
\hline Descending colon & $31(19.1)$ \\
\hline Sigmoid colon & $24(14.8)$ \\
\hline Rectum & $74(45.7)$ \\
\hline \multicolumn{2}{|l|}{ Histological classification, $n(\%)$ : } \\
\hline Tubular adenoma & $63(38.9)$ \\
\hline Tubulovillous adenoma: & $28(17.3)$ \\
\hline Villous adenoma & $34(21.0)$ \\
\hline HGIN & $24(14.8)$ \\
\hline Submucosal carcinoma & $1(0.6)$ \\
\hline Serrated polyp & $3(1.8)$ \\
\hline Inflammatory polyp & $9(5.6)$ \\
\hline Procedure duration, mean (range) [min] & $56(12-135)$ \\
\hline En bloc resection, $n(\%)$ & $161(99.3)$ \\
\hline \multicolumn{2}{|l|}{ Complication, $n(\%)$ : } \\
\hline Bleeding & $1(0.6)$ \\
\hline Perforation & $1(0.6)$ \\
\hline Recurrence, $n$ (\%) & $0(0)$ \\
\hline Total & 162 \\
\hline
\end{tabular}

ESD - endoscopic submucosal dissection, LST - laterally spreading tumor HGIN - high-grade intraepithelial neoplasia. was 53.9 years (range: $24-81$ years), with a male to female ratio of $1.35: 1$ (93/69). The mean diameter of lesions was $46 \mathrm{~mm}(12-100 \mathrm{~mm})$, including 125 cases that were more than $20 \mathrm{~mm}$ in diameter and 37 cases that were less than $20 \mathrm{~mm}$. The distribution of LST subtypes was 61 LST-G-H, 57 LST-G-M, 39 LST-NG-F, and 5 LST-NG-PD. Overall, the most common location was the rectum $(74,45.7 \%)$, followed by the descending colon $(31,19.1 \%)$, the sigmoid colon $(24,14.8 \%)$, the transverse colon $(17,10.5 \%)$, the ascending colon $(11,6.8 \%)$ and cecum $(5,3.1 \%)$. The most common histological type was tubular adenoma with 63 (38.9\%) cases, followed by villous adenoma with 34 (21.0\%) cases, tubulovillous adenoma with 28 (17.3\%) cases, high-grade intraepithelial neoplasia (HGIN) with 24 (14.8\%) cases, inflammatory polyp with 9 (5.6\%) cases, serrated polyp with $3(1.8 \%)$ cases, and submucosal invasive carcinoma with $1(0.6 \%)$ case (additional radical procedures were reserved for them) (Table I). In 161 patients, we obtained complete dissection of the LST by using the Dual knife and the complete histological evaluation. The mean operating time was 56 min (range: 12-135 min) (Table I). One perforation (0.6\%) with $3 \mathrm{~mm} \times 3 \mathrm{~mm}$ occurred during the procedure, and the patient recovered after being treated with endoscopic metal clips. One case of delayed bleeding $(0.6 \%)$ was observed on postoperative day (POD) 3 , and the patient underwent endoscopic clipping and achieved successful hemostasis. Blood transfusion was not required for the patient. No case of local persistence and recurrence or metastasis was observed at a follow-up endoscopy 6 to 31 months after the en bloc resection.

\section{Discussion}

Colorectal LSTs are classified into LST-Gs and LSTNGs, according to macroscopic findings, which are an important kind of precursor lesions of colorectal carcinoma. Furthermore, the carcinoma incidence rate and the submucosal invasion rate were reported to be higher in LST-NG than in LST-G [4]. Thus, we recognize that morphological classification for LST is significant for planning appropriate treatment strategies for patients. The EMR, piecemeal EMR, or ESD for such LSTs is an effective treatment. Piecemeal EMR is associated with higher local recurrence rates, ranging from $10 \%$ to $23.5 \%$, as compared with EMR, whose local recurrence rates vary from $0 \%$ to $9.1 \%$ 
for en bloc resections, and from $0 \%$ to $3 \%$ following ESD [16-18]. Moreover, LST-NGs and residual or locally recurrent lesions (after prior endoscopic treatment) are considered technically difficult to treat because of the presence of submucosal fibrosis. Consequently, for colorectal LSTs, especially larger than $20 \mathrm{~mm}$ in diameter, ESD is a greatly appropriate choice that enables the en bloc resection regardless of the size of the lesion, accurate histological evaluation, and a reduced possibility of residual lesions or local recurrence.

The ESD has been the first-line endoscopic treatment for superficial gastrointestinal tract tumors [19]. The devices applied in ESD are crucial for performing the procedure successfully. Nowadays, a large number of knives have been developed for ESD. Representative knives that are frequently applied in colorectal ESD are as follows: Hook knife, IT knife, Flush knife, Flex knife, Dual knife, SB knife, B-knife, and Triangle tip knife. Of these, the Dual knife is one of the most constantly used knives and has some advantageous functions including incision, dissection, and hemostasis. The knife is equipped with a minute disk tip and lengths of $1.5 \mathrm{~mm}$ and $2 \mathrm{~mm}$. Currently, there are 3 types of Dual knife: KD-650Q, KD-650U, and KD-650L. Of these, the KD-650Q is usually used for ESD of colorectal tumors with a disk tip (the length of $1.5 \mathrm{~mm}$ ). Then the knife with a length of $1.5 \mathrm{~mm}$ is usually used for ESD of colorectal tumors. When small blood vessels are exposed or bleeding occurs during the ESD procedures, colonoscopists can usually prevent bleeding or stop bleeding successfully with the electrocoagulation mode of the Dual knife, instead of changing hemostatic devices. Furthermore, it can keep the endoscopic views clear, avoid complications, and decrease the procedure time. For large vessels or small vessels that cannot achieve hemostasis, hemostatic forceps or endoscopic metal clips should be used.

In our study, we reported our encouraging experience of ESD by using the Dual knife only for colorectal LSTs. Thereby we summarized some technical skills of application of the Dual knife during the ESD procedure on the basis of our experiences with it as follows: 1) Keep an appropriate working distance between the tip of the knife and the tip of the endoscope, then the tip of the knife is pressed softly on the mucosa and the cutting line is under the direct visualization during mucosal cutting, avoiding the blind operation. 2) Control appropriate tension with regard to mucosal thickness, tumor location, and degree of mucosa prone to bleeding during mucosal incision. For lesions located in the rectum with thick mucosa, we can increase properly the incising tension and power setting. On the other hand, for mucosa prone to bleeding or thin mucosa, tension and power setting are decreased to prevent deep incising. 3) During submucosal dissection, the thickness of the submucosal cushion should be maintained continuously. As the incision proceeds, dissection is carried out. Dissecting submucosa of a lesion is better performed in the longitudinal direction with the aid of gravity or the transparent cap, avoiding damage to the muscular layer. 4) Mucosal incision and submucosal dissection are sometimes very difficult during the final stage of ESD procedures. Therefore, we ought to adjust the position and direction of the tip of the Dual knife and focus on the target tissue, avoiding damage to the surrounding mucosa.

For these recent cases, all the colorectal ESD was completed with the Dual knife, which reduces the impact of exchanging attachments on the operation time. Thus, utilization of the Dual knife for colorectal ESD is worthy of clinical widespread application. Furthermore, expert endoscopists are essential for carrying out high-quality ESD with the Dual knife. As with all the other new technologies, a learning curve exists in the process of ESD [20-22]. Systematic teaching within gastroenterology training procedures, intensive short courses, advanced endoscopy equipment, and computer-based training patterns may assist inexperienced endoscopists to learn endoscopic techniques.

Our experience was limited by the fact that it was a retrospective study that was carried out at a single hospital with a finite number of patients. Apart from that, diverse therapeutic instruments and procedures (injection solutions, coagulation models, etc.) would contribute to increased variability in the observed outcomes.

\section{Conclusions}

With regard to the colorectal LSTs, ESD using the Dual knife is safe, feasible, and minimally invasive. However, to confirm our results, larger trials with a longer follow-up are required together with improvement of the technique and of the technical devices. A prospective multicenter trial is needed to 
confirm the usefulness of the Dual knife in comparison to other ESD knives.

\section{Acknowledgments}

We are sincerely grateful to the medical staff in our endoscopy center for their collaboration on this work.

\section{Conflict of interest}

\section{The authors declare no conflict of interest.}

\section{References}

1. Kudo S. Endoscopic mucosal resection of flat and depressed types of early colorectal cancer. Endoscopy 1993; 25: 455-61.

2. Hiraoka S, Kato J, Tatsukawa M, et al. Laterally spreading type of colorectal adenoma exhibits a unique methylation phenotype and K-ras, mutations. Gastroenterology 2006; 131: 379-89.

3. Kudo Se, Lambert R, Allen JI, et al. Nonpolypoid neoplastic lesions of the colorectal mucosa. Gastrointest Endosc 2008; 68 (4 Suppl): S3-47.

4. Uraoka T, Saito Y, Matsuda T, et al. Endoscopic indications for endoscopic mucosal resection of laterally spreading tumours in the colorectum. Gut 2006; 55: 1592-7.

5. Tanaka S, Kashida H, Saito Y, et al. JGES guidelines for colorectal endoscopic submucosal dissection/endoscopic mucosal resection. Dig Endosc 2015; 27: 417-34.

6. Oyama T, Tomori A, Hotta K, et al. Endoscopic submucosal dissection of early esophageal cancer. Clin Gastroenterol Hepatol 2005; 3 (7 Suppl 1): S67-70.

7. Gotoda T, Kondo H, Ono H, et al. A new endoscopic mucosal resection procedure using an insulation-tipped electrosurgical knife for rectal flat lesions: report of two cases. Gastrointest Endosc 1999; 50: 560-3.

8. Toyanaga T, Man IM, Ivanov D, et al. The results and limitations of endoscopic submucosal dissection for colorectal tumors. Acta Chir lugosl 2008; 55: 17-23.

9. Kodashima S, Fujishiro M, Yahagi N, et al. Endoscopic submucosal dissection using flexknife. J Clin Gastroenterol 2006; 40: 378-84.

10. Sano Y, Fu KI, Saito Y, et al. A newly developed bipolar-current needle-knife for endoscopic submucosal dissection of large colorectal tumors. Endoscopy 2006; 38 Suppl 2: E95.

11. Battaglia G, Antonello A, Realdon S, et al. Flexible endoscopic treatment for Zenker's diverticulum with the SB Knife. Preliminary results from a single-center experience. Dig Endosc 2015. 27: 728-33.

12. Nishizawa T, Yahagi N. Endoscopic mucosal resection and endoscopic submucosal dissection: technique and new directions. Curr Opin Gastroenterol 2017; 33: 315-9.

13. Lai E J, Calderwood A H, Doros G. The Boston bowel preparation scale: a valid and reliable instrument for colonoscopy-oriented research. Gastrointest Endosc 2009; 69: 620-5.

14. Fujimoto A, Uraoka T, Nishizawa T, et al. Rebamipide solution: a novel submucosal injection material to promote healing speed and healing quality of ulcers induced by endoscopic submucosal dissection. Gastrointest Endosc 2018; 87: 1114-20.

15. Schlemper RJ, Riddell RH, Kato Y, et al. The Vienna classification of gastrointestinal epithelial neoplasia. Gut 2000; 47: 251-5.

16. Fujiya M, Tanaka K, Dokoshi T, et al. Efficacy and adverse events of EMR and endoscopic submucosal dissection for the treatment of colon neoplasms: a meta-analysis of studies comparing EMR and endoscopic submucosal dissection. Gastrointest Endosc 2015; 81: 583-95.

17. De Ceglie A, Hassan C, Mangiavillano B, et al. Endoscopic mucosal resection and endoscopic submucosal dissection for colorectal lesions: a systematic review. Crit Rev Oncol Hematol 2016; 104: 138-55.

18. Oka S, Tanaka S, Saito Y, et al. Local recurrence after endoscopic resection for large colorectal neoplasia: a multicenter prospective study in Japan. Am J Gastroenterol 2015; 110: 697-707.

19. Draganov PV, Wang AY, Othman MO, et al. AGA Institute Clinical Practice Update: Endoscopic submucosal dissection in the United States. Clin Gastroenterol Hepatol 2019; 17: 16-25.

20. Sato R, Fujiya M, Watari J, et al. The diagnostic accuracy of high-resolution endoscopy, autofluorescence imaging and narrow-band imaging for differentially diagnosing colon adenoma. Endoscopy 2011; 43: 862-8.

21. Rogart JN, Jain D, Siddiqui UD, et al. Narrow-band imaging without high magnification to differentiate polyps during real-time colonoscopy: improvement with experience. Gastrointest Endosc 2008; 68: 1136-45.

22. Rastogi A, Pondugula K, Bansal A, et al. Recognition of surface mucosal and vascular patterns of colon polyps by using narrow-band imaging: Interobserver and intraobserver agreement and prediction of polyp histology. Gastrointest Endosc 2009; 69: $716-22$.

Received: 20.02.2019, accepted: 10.03.2019. 